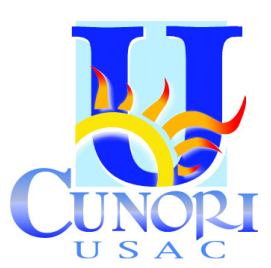

\author{
Revista Ciencia Multidisciplinaria CUNORI \\ http://revistacunori.com \\ DOI: https://doi.org/10.36314/cunori.v2i1.46 \\ ISSN: 2617- 474X (impresa) / 2617- 4758 (en línea)
}

Como citar el artículo

Cuellar, K., Retana, R. \& Mazariegos, E. (2018). Caracterización del patrón alimenticio en niños menores de un año de edad. Revista Ciencia Multidisciplinaria CUNORI, 2(1), 7-16. https://doi.org/10.36314/cunori.v2i1.46

\title{
Caracterización del patrón alimenticio en niños menores de un año de edad
}

\section{Characterization of the alimentary pattern in children younger than one year old}

\author{
Katherine Cuéllar *, Ronaldo Retana \& Edvin Mazariegos \\ Centro Universitario de Oriente CUNORI, Universidad de San Carlos de Guatemala \\ Recibido: 01 de abril de 2018 Aceptado: 31 de julio de 2018 \\ Disponible en internet el 17 de agosto de 2018
}

*Autor para correspondencia.

Correo electrónico: katyalas.92@gmail.com

Resumen

undialmente se ha estimado que el 34.8\% de los lactantes reciben lactancia materna exclusiva (LME) durante los primeros

16 meses de vida y que los alimentos complementarios son introducidos demasiado temprano o demasiado tarde y son en general, nutricionalmente inadecuados e inseguros. El propósito es caracterizar el patrón alimenticio en niños menores de 1 año de edad en base al conocimiento de las madres que acuden a la consulta externa del Hospital Nacional de Chiquimula. La LME óptima podría evitar, globalmente, el 13\% de muertes en niños menores de 5 años y prácticas apropiadas de alimentación complementaria y lograr un beneficio adicional del 6\% en la reducción de la mortalidad de los menores de 5 años. El estudio es descriptivo transversal, el cual se realizó encuestando de manera verbal a 288 madres de niños menores de 1 año de edad que acudieron a la consulta externa del Hospital Nacional de Chiquimula. De 288 niños menores de 1 año de edad, 200 (69.4\%) recibieron LME: 150 $(68.8 \%)$ del grupo de menores o igual a 6 meses y 50 (71.4\%) del grupo de 7 a menores de 12 meses en el momento de la encuesta. La principal razón por la que no se cumplió con LME fue en un 30.6\% por escasa o nula producción de leche. La edad de inicio de la alimentación complementaria fue a los 6 meses con $55.05 \%$ y el principal grupo alimenticio utilizado para la ablactación fue tubérculos con $77.1 \%$.

Palabras clave: lactancia materna exclusiva, alimentación mixta, fórmula exclusiva de inicio, ablactación, patrón alimenticio

Abstract

$\mathrm{W}$

orldwide it is estimated that $34.8 \%$ of the infants receive breastfeeding exclusively (LME) during the first six months of life and the supplementary food are introduced too early or too late and are general nutritionally inadequate and insecure. Characterization of the alimentary pattern in children younger than one year old on the base of the mother's knowledge of the outpatients that come to the National Hospital of Chiquimula. The optimum LME could prevent, worldwide the $13 \%$ of deaths in children younger than 5 years old and appropriate supplementary feeding practices could have additional benefit of $6 \%$ in the reduction of mortality in children younger than 5 years old. Descriptive cross-sectional study, where verbally polled 288 mothers of children under one year old, that went to external consultations of the National Hospital of Chiquimula. Of 288 children younger than one year old, $200(69.4 \%)$ got LME: $150(68.8 \%)$ of the group of 6 months or younger and $50(71.4 \%)$ of the group of 7 months to 12 months in the moment of the survey. The main reason why it wasn't fulfilled the LME was a 30.6\% for little or no milk production. The age of the beginning of the supplementary feeding was at six months with $55.05 \%$ and the main feeding group used for the ablation were tubercles with $77.1 \%$.

Los textos publicados en la revista son responsabilidad exclusiva de los autores. 
Keywords: exclusive maternal breastfeeding, mix alimentation, exclusive formula from the beginning, ablation, alimen tary patterns

\section{Introducción}

Las recomendaciones del Fondo de las Naciones Unidas para la Infancia (UNICEF) y la Organización Mundial de la Salud (OMS) para una alimentación infantil óptima, tal como se encuentran establecidas en la "Estrategia Mundial para alimentación del lactante y del niño pequeño" es la lactancia materna exclusiva durante los primeros 6 meses de vida e iniciar alimentación complementaria, adecuada y segura, a partir de los 6 meses de edad, manteniendo la lactancia materna hasta los dos años de edad o más (OMS y OPS, 2010).

Se ha estimado que las prácticas inadecuadas de lactancia materna (LM) y no practicar especialmente la lactancia materna exclusiva (LME) durante los primeros seis meses de vida, provoca 1.4 millones de muertes y el 10\% de la "carga" de enfermedades entre los niños menores de 5 años (OMS y OPS, 2010). En los primeros años de vida, los niños y niñas representan una gran demanda nutricional por su velocidad de crecimiento, requerimientos nutricionales y su capacidad de defenderse contra enfermedades (UNICEF, 2012).

Debido al crecimiento del lactante, a partir de los seis meses de edad se genera un déficit de energía que la leche materna no logra cubrir, este déficit se conoce como Brecha de Energía (BE), a mayor edad, mayor deberá ser el aporte energético de la alimentación complementaria (AC), mientras que se hace menor el aporte energético de la LM. Al introducir la AC en la dieta del lactante, éste tiende a disminuir la ingesta de LM, hasta que progresiva y gradualmente la AC desplaza la LM (INCAP, s.f.).

En el lactante de 6 a 12 meses de edad se debe continuar la LM al mismo tiempo que se introduce la AC porque la LM aporta el 50\% o más de los requerimientos de energía y nutrientes del niño que se encuentra en este rango de edad y aproximadamente el 30\% entre los 12 a 24 meses de edad. La LM sigue aportando energía y nutrientes de buena calidad (aunque sea en menor cantidad) mientras el niño comienza a recibir los alimentos complementarios, los cuales darán un aporte adicional de energía y nutrientes, así mismo que la LM aún continuará siendo una fuente importante de alimentación cuando el niño de 6 a 12 meses presente alguna enfermedad y será un protector de la desnutrición en el lactante (INCAP, s.f.).

Tanto los alimentos como las prácticas de alimentación influyen sobre la calidad de la alimentación complementaria y las madres y las familias necesitan apoyo para aplicar buenas prácticas de alimentación complementaria (OMS y OPS, 2010).

\section{Materiales y métodos}

Se tomó de muestra a 288 madres de niños menores de un año de edad que acudieron a la consulta externa del Hospital Nacional de Chiquimula, procedentes del departamento de Chiquimula, para la cual se realizó una proyección de los meses de octubre, noviembre y diciembre de 2017 para la obtención del universo, el cual fue de 1,839 consultas, aplicándosele entonces la fórmula de tamaño de la muestra 
por distribución directa. Para realizar la caracterización del patrón alimenticio se realizó una encuesta a las madres, la cual fue de elaboración propia, realizada de forma verbal por parte de la encuestadora, para facilitar y agilizar la aplicación de la misma, ya que algunas de las madres que acudieron a la consulta externa no poseen el nivel educativo necesario para leer y escribir, ésta se realizó únicamente a las madres que acudieron a la consulta externa del Hospital Nacional de Chiquimula y cuyos niños fueran menores de un año de edad, así mismo, se excluyeron a todas las madres que no pertenecieran al departamento de Chiquimula.

La encuesta se divide en 3 apartados: datos generales de la madre (edad, sexo, religión, ocupación, entre otros), datos del niño (edad), seguido así mismo, de 18 preguntas de opción múltiple para medición de conocimiento que poseen las madres de manera general sobre el tema de lactancia materna y ablactación. Con base en la encuesta que se utilizó para la recolección de datos, se procedió a ordenar de acuerdo a las variables, creando una base de datos en Epilnfo 7. Al finalizar la recolección de datos, se crearon cuadros y figuras, para representar los datos obtenidos a través de la investigación, con frecuencias simples y porcentajes.

\section{Resultados}

\section{Edad de niños menores de un año de edad}

De acuerdo a la edad de los niños menores de un año que acudieron a la consulta externa del Hospital Nacional de Chiquimula con sus madres al momento de realizar la encuesta, el rango de edad que muestra mayor número es el de menores o igual a 6 meses con 218 niños (75.7\%); el grupo de niños de 7 a menores de 12 meses fueron 70, concretamente, de 7 a 9 meses un total de 42 (14.6\%) y de 10 a menores de 12 meses 28 niños $(9.7 \%)$.

\begin{tabular}{|c|c|c|c|}
\hline \multicolumn{2}{|c|}{ Edad } & Frecuencia & Porcentaje (\%) \\
\hline \multicolumn{2}{|c|}{ Niños-as $\leq 6$ meses } & 218 & $75.70 \%$ \\
\hline \multirow{2}{*}{$\begin{array}{l}\text { Niños-as } \\
>6 \text { meses }\end{array}$} & 7 a 9 meses & 42 & $14.60 \%$ \\
\hline & $\begin{array}{c}10 \mathrm{a}<12 \\
\text { meses }\end{array}$ & 28 & $9.70 \%$ \\
\hline \multicolumn{2}{|c|}{ Total } & 288 & $100 \%$ \\
\hline
\end{tabular}

Cuadro 1. Distribución según edad de niños menores de un año de edad que acudieron con sus madres a la consulta externa del Hospital Nacional de Chiquimula de octubre a noviembre de 2017. 
Prevalencia de alimentación con lactancia materna exclusiva (LME), fórmula exclusiva y alimentación mixta

De 288 niños menores de un año de edad 69.4\% (200) de ellos recibió LME: 68.8\% (150) comprendidos en el rango de menores o igual a 6 meses y 71.4\% (50) del grupo de 7 a menores de 12 meses. En lo que respecta a los 30.6\% (88) niños restantes, 68 fueron alimentados con alimentación mixta y 20 con fórmula exclusiva de inicio, quedando exentos de recibir los beneficios y nutrientes que la lactancia materna ofrece.

\begin{tabular}{|c|c|c|c|c|c|c|}
\hline \multicolumn{2}{|c|}{$\begin{array}{l}\text { Edad/ } \\
\text { Patrón alimenticio }\end{array}$} & $\begin{array}{l}\text { Niños-as } \\
\leq 6 \text { meses }\end{array}$ & $\%$ & $\begin{array}{c}\text { Niños-as de } \\
7 \mathrm{a}<12 \text { meses }\end{array}$ & $\%$ & Total \\
\hline \multicolumn{2}{|c|}{ Recibieron } & 150 & 68.8 & 50 & 71.4 & 200 \\
\hline \multirow{2}{*}{$\begin{array}{l}\text { No recibieron } \\
\qquad \mathrm{LME}\end{array}$} & $\begin{array}{l}\text { Alimentación } \\
\text { mixta }\end{array}$ & 54 & 24.8 & 14 & 20 & 68 \\
\hline & $\begin{array}{l}\text { Fórmula } \\
\text { exclusiva }\end{array}$ & 14 & 6.4 & 6 & 8.6 & 20 \\
\hline \multicolumn{2}{|c|}{ Subtotal } & 68 & 31.2 & 20 & 28.6 & 88 \\
\hline \multicolumn{2}{|c|}{ Total } & 218 & 100 & 70 & 100 & 288 \\
\hline
\end{tabular}

Cuadro 2. Prevalencia de LME, prevalencia de la alimentación con fórmula exclusiva (de inicio) y alimentación mixta en niños menores de un año de edad que acudieron a la consulta externa del Hospital Nacional de Chiquimula de octubre a noviembre de 2017.

Razones más frecuentes de suspensión de la lactancia materna exclusiva (LME) en niños menores o iguales a 6 meses

Entre las principales razones por las cuales las madres suspendieron la LME en sus hijos menores o iguales a 6 meses fueron: escasa o nula producción de leche con 30.6\% (27), por su profesión u oficio y sentimiento de que el bebé no se saciaba, ambas en segundo lugar con 17.1\% (15) y en tercer lugar por razones personales con $10.2 \%(9)$. 


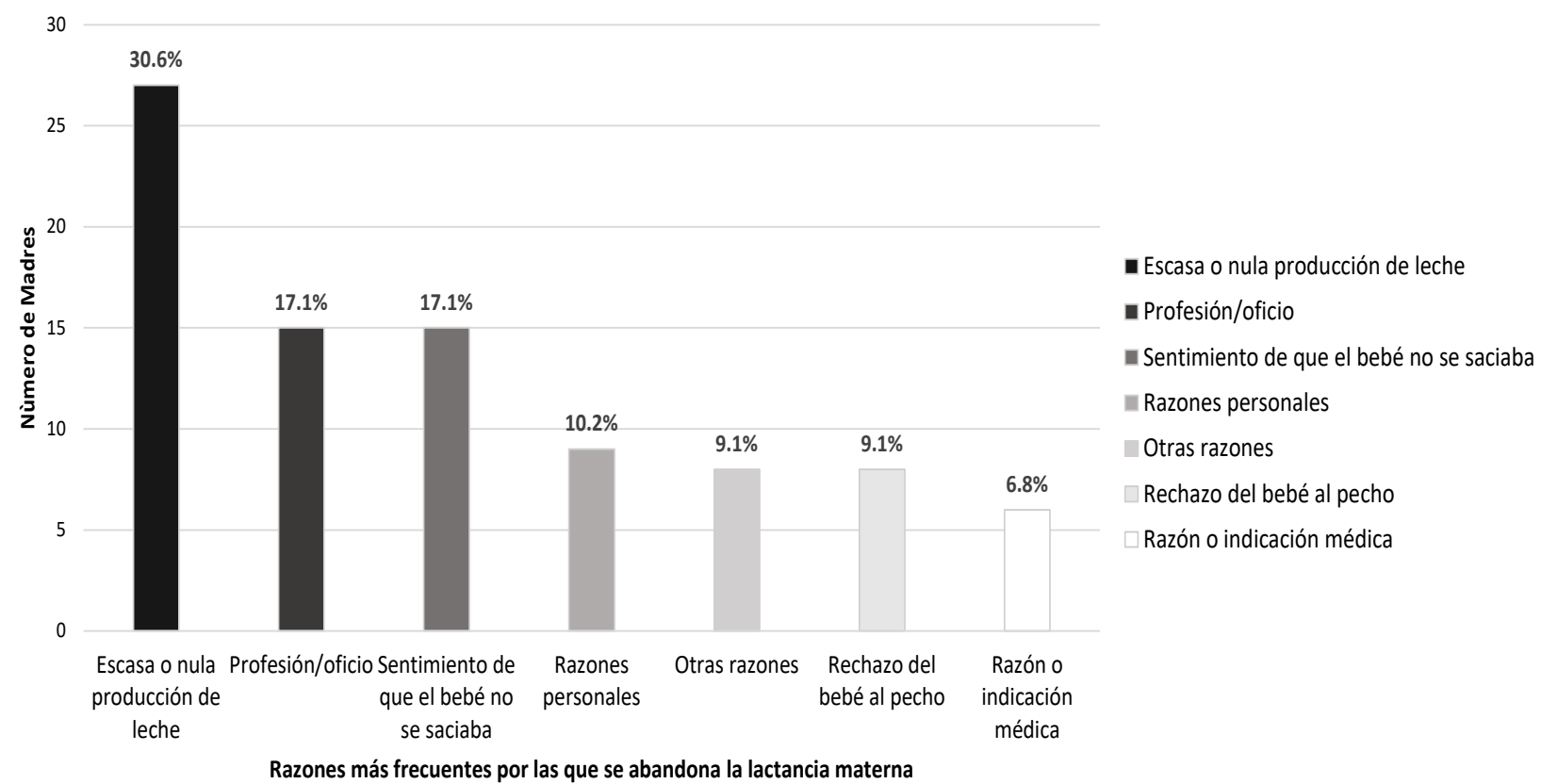

Figura 1. Razones más frecuentes por las cuales las madres suspenden la alimentación de niños menores o igual 6 meses con lactancia materna exclusiva que acudieron a la consulta externa del Hospital Nacional de Chiquimula de octubre a noviembre de 2017.

\section{Ablactación}

Al momento de realizar la investigación y encuestar a las 288 madres, se les preguntó si en ese momento ellas estaban dando ablactación a sus hijos, para lo cual había la opción de sí y no. Un 37.8\% (109) madres si estaban dando alimentación complementaria o ablactación a su hijo al momento de la investigación y el restante $62.2 \%$ (179) no lo había puesto en práctica aún.

\begin{tabular}{lcc}
\hline $\begin{array}{l}\text { Madres que han iniciado alimentación complementaria al } \\
\text { momento de la encuesta }\end{array}$ & Frecuencia & Porcentaje (\%) \\
\hline $\mathrm{Si}$ & 109 & $37.80 \%$ \\
$\mathrm{No}$ & 179 & $62.20 \%$ \\
Total & 288 & $100 \%$ \\
\hline
\end{tabular}

Cuadro 3. Distribución de madres que han iniciado alimentación complementaria (AC) o ablactación al momento de la encuesta, en niños menores de un año de edad que acudieron con sus madres a la consulta externa del Hospital Nacional de Chiquimula de octubre a noviembre de 2017.

La edad de inicio de la alimentación complementaria fue con un 55.05\% (60) la de los 6 meses de edad y los tres principales grupos alimenticios utilizados para ésta fueron: tubérculos con $77.1 \%$ (222), cereales $61.5 \%$ (177) y verduras con $51.0 \%$ (147). 
Edad a la que se inició $A C$ en los niños que al momento de la encuesta Sí la utilizan

Edad (meses)

Frecuencia

Frecuencia
Porcentaje

(\%)

Edad a la que se iniciará AC en los niños que al momento de la encuesta NO la utilizan

Edad (meses)

Frecuencia

Porcentaje

(\%)

\footnotetext{
*AC: Alimentación complementaria
}

Cuadro 4. Distribución de acuerdo a la edad de inicio de alimentación complementaria (AC) o ablactación en niños menores de un año de edad que acudieron con sus madres a la consulta externa del Hospital Nacional de Chiquimula de octubre a noviembre de 2017.

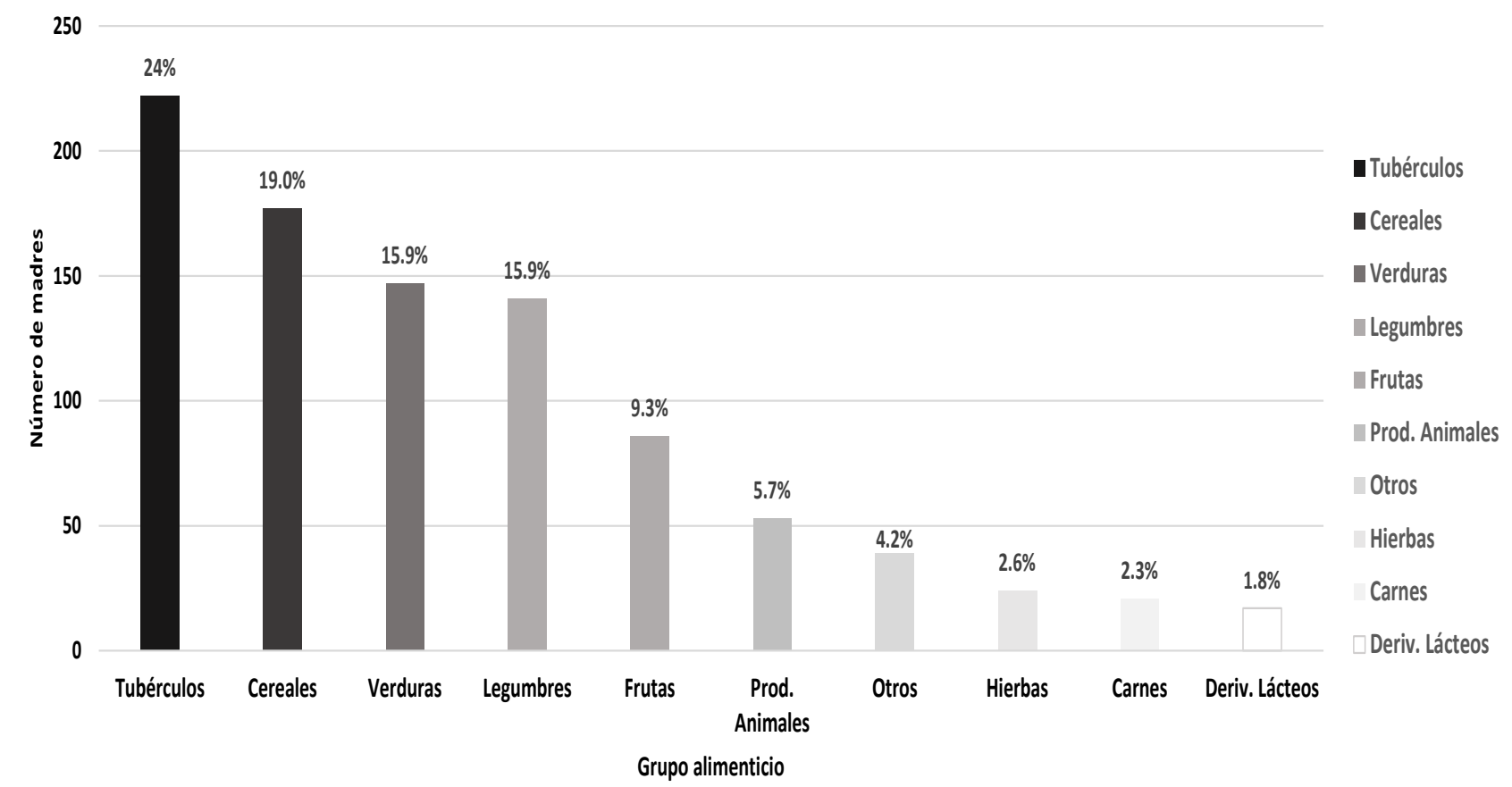

Figura 2. Frecuencias y porcentajes de los grupos de alimentos más utilizados para el inicio de ablactación por las madres de niños menores de un año de edad que acudieron a la consulta externa del Hospital Nacional de Chiquimula de octubre a noviembre de 2017.

Se muestra el grupo de alimentos más utilizados por las madres en los niños menores de un año, siendo los tubérculos con un $24 \%$ y los cereales con $19 \%$ los alimentos que más utilizan las madres. 


\section{Discusión}

Se realizó un estudio descriptivo transversal en la consulta externa del Hospital Nacional de Chiquimula, con el cual, se buscaba poder caracterizar el patrón alimenticio de niños menores de un año de edad que acudieran con sus madres durante los meses de octubre a noviembre de 2017. Las recomendaciones para una alimentación óptima y adecuada para todo lactante por parte de la OMS y UNICEF es que éste debe ser alimentado de manera exclusiva los primeros 6 meses de su vida con lactancia materna exclusiva y luego complementarla a partir de los 6 meses de edad con la introducción de alimentos (alimentación complementaria), continuando la lactancia materna hasta los dos años de vida o más. En lo que respecta a los 288 niños menores de un año de edad que acudieron con sus madres, 218 de ellos se encontraban en el rango de menores o igual a 6 meses de edad y 70 en el rango de 7 a menores de 12 meses de edad. De esos 218 niños menores o igual a 6 meses, 150 recibían lactancia materna al momento de ser realizada la investigación y 50 niños del rango de 7 a menores de 12 meses recibió LME en los primeros seis meses de su vida; en total 200 (69.4\%) niños menores de un año de edad recibieron LME.

Los niños alimentados con alimentación mixta (lactancia materna más fórmula) y alimentación con fórmula exclusiva, de los 218 niños que corresponden al grupo de menores o igual a 6 meses, el $24.8 \%$ (54) recibía alimentación mixta y el 6.4\% (14) fórmula exclusiva, por tanto, el 31.2\% (68) niños menores o igual a 6 meses no recibía LME y de los 70 niños del grupo de edad de 7 a menores de 12 meses, 28.6\% (20) no recibió LME en sus primeros 6 meses de vida, por lo que sus madres la sustituyeron con alimentación mixta en un $20 \%$ (14) y con fórmula exclusiva en un 8.6\% (6).Un total de 88 (30.6\%) niños menores de un año de edad no recibió LME, lo que los deja exentos de recibir las principales vitaminas, nutrientes y defensas de enfermedades más frecuentes a esta edad.

Factores como la escasa o nula producción de leche materna al momento del nacimiento del bebé y en los primeros días posteriores al mismo, conlleva a la madre el sustituir parcial o completamente el pecho por algún sucedáneo de la leche materna (fórmula). Este factor es frecuente ya que en la mayoría de las mujeres la producción de la leche materna aumenta lentamente según las necesidades del bebé y también puede variar la cantidad de la misma durante el día, es entonces cuando el niño o la niña puede que demande el pecho con mayor frecuencia y la madre creer que no tiene la suficiente cantidad de leche. Además en muchas ocasiones, las madres sufren preocupaciones y ansiedad en relación con la cantidad de leche que puedan tener, ya que las hormonas encargadas de provocar la producción y eyección de la leche (oxitocina y prolactina) pueden estar influenciadas por el estado de ánimo de la madre, si se siente nerviosa, incómoda, insegura, estas hormonas pueden dejar de producirse y disminuye la cantidad y salida de la leche, lo contrario ocurrirá si se siente apoyada, protegida y segura.

Es necesario entonces que la preparación y consejería acerca de la lactancia materna no sea exclusiva para las madres, sino también para familiares y miembros de la comunidad.Otros factores influyentes son el trabajo u oficio, ya que en muchos de ellos no les dan disponibilidad de horario para poder dar de lactar, por lo que las madres se ven en la dificultad de no poder alimentar a su bebé con lactancia materna en su totalidad. La sensación de que el bebé no queda completamente saciado siendo alimentado únicamente con lactancia fue otra de las razones por las cuales las madres no cumplieron con lactancia materna exclusiva, esto se debe a que las madres observan que el bebé llora mucho aun cuando ya han sido alimentados o que piden pecho en un intervalo corto de tiempo luego de la última mamada. Sin embargo, entre los consejos de la OMS y UNICEF para una lactancia materna exclusiva, se menciona 
el dar lactancia materna en forma de libre demanda al niño/niña, es decir, el bebé debe ser alimentado cada vez que quiera. Esto significa que el horario no es estricto, aunque puede ser cada 2 o 3 horas, de día y noche, los primeros 30 días y luego, progresivamente, cada 3 a 4 horas hacia finales del segundo mes. La duración de cada mamada varía entre 5 a 15 minutos en cada pecho y debe dársele suficiente tiempo al bebé ya que cada uno es diferente.

Los motivos personales como el no querer alimentar a su hijo a través del seno materno, rechazo del bebé al pecho, indicación médica y otros motivos como el no estar todo el tiempo en casa por lo que otra persona estaría con el bebé cuidándolo; y dolor en los pechos al amamantar, fueron las razones menos frecuentes por las cuales las madres no cumplían con la lactancia materna exclusiva en la alimentación de sus hijos. De la población en estudio el 37.8\% de las madres practicaban la alimentación complementaria al ser encuestadas; el 55\% (60) de ellas había iniciado esta práctica a la edad correcta (6 meses), el 13.8\% (15) la inició después de los 6 meses y un 10\% (11) la había iniciado de manera precoz a los 3 meses. Así mismo, de las $62.2 \%$ que aún no practicaban la alimentación complementaria afirmaron que iniciarían esta práctica cuando su niño/niña cumpliera los 6 meses de edad, el 9\% (16) lo haría después de los 6 meses y el 5\% (9) dijo que lo haría entre los 4 o 5 meses de edad.

La OMS recomienda el inicio de la alimentación complementaria a partir de los 6 meses por diversas razones. A partir de esta edad el lactante ya ha logrado un desarrollo adecuado de su función digestiva, renal y de la maduración neurológica, sin olvidar que, la lactancia materna ya no es suficiente para poder cubrir las necesidades nutricionales y energéticas del niño. Cuando se inicia la alimentación de forma precoz (antes de los 6 meses), se deja al niño/niña expuesto a sufrir alergias alimenticias debido a que su aparato digestivo no está totalmente maduro y por lo tanto las paredes intestinales son permeables, por lo cual, las alergias pueden afectar cualquier sistema del cuerpo provocando náuseas, vómitos, diarreas y estreñimiento, razones que muchas veces son el motivo de consulta al médico. Por otro lado, el inicio de la alimentación complementaria de forma tardía tampoco es una buena decisión, ya que entre los 6 a 23 meses hay un mayor pico de incidencia de retraso en el crecimiento, deficiencias de micronutrientes y enfermedades infecciosas.

Los alimentos más utilizados por las madres para alimentar a sus hijos son la papa (tubérculos), pan desecho en café, "Nestum", "Incaparina" (cereales), zanahoria, güisquil (verduras), caldo de frijol (legumbres), banano, manzana (frutas), esto debido a que son alimentos que las madres consideran apropiados para la edad del bebé y son accesibles económicamente para la mayoría de ellas; a diferencia del huevo (productos animales), compotas (otros), caldo de hierba mora y chipilín (hierbas), carne de pollo, res, hígado (carnes) y derivados lácteos como leche, queso y requesón, que fueron los menos utilizados ya que las madres consideran que éstos son más apropiados para una edad mayor, entre $10 \mathrm{y}$ 12 meses que para el inicio de la alimentación complementaria a los 6 meses, además de que muchas madres señalaron que muchas veces no incluían algunos de los alimentos mencionados ya que no había disponibilidad económica para consumirlos. Algunos alimentos que se utilizan muchas veces como alimento para los niños no son nutricionalmente adecuados, como lo son los caldos y el café. Guías alimenticias para lactantes recomiendan evitar los caldos o sopas porque al ser preparaciones con mucha agua y poco valor nutricional el volumen que se requiere es muy grande para cubrir las necesidades nutricionales del niño/niña, por lo cual se recomienda mejor triturar o moler los alimentos para evitar la dilución innecesaria. 
En lo que al café se refiere, éste contiene calorías que no proporcionan ningún nutriente por lo cual los niños que se llenan con este tipo de bebidas no obtienen las vitaminas ni minerales que necesitan, además de que la cafeína evita que reciban el calcio necesario para desarrollar unos dientes y huesos fuertes. Otra razón por la cual no se recomienda el consumo de cafeína en la dieta de los niños es porque funciona como diurético por lo cual puede contribuir a la deshidratación. Todo lo que se determinó en función de las madres realizaban respecto a la alimentación de su bebé estuvo mayormente influenciado por consejos o directrices que recibieron de personas cercanas a ellas como lo eran sus madres y suegras (miembros de la familia) ya que estás son las personas a las que tienen mayor confianza y que ellas consideran tienen el conocimiento por ser personas mayores con experiencia. Personal de centro o puesto de salud y la propia experiencia de la madre, fueron factores determinantes para la alimentación de los niños y la menor influencia la obtuvieron del personal del hospital, pediatra, otras madres con experiencia, medios de comunicación, internet y vecinas.

La información obtenida del estudio es positiva y alentadora, ya que muestra en forma general que las madres, si tienen el conocimiento adecuado y que por lo tanto la mayoría de ellas alimenta adecuadamente a sus hijos. Sin embargo, esto no quiere decir que no haya madres que aún no conocen cómo debe ser la dieta de sus niños, lo que muchas veces conlleva a que haya desnutrición, disminución del crecimiento y desarrollo, como también alergias alimenticias y obesidad en un período de largo plazo. Por lo que es de importancia buscar una mayor comunicación del tema y así orientar a las madres para que los errores que se cometen ahora puedan ir disminuyendo en un futuro hasta el punto de que éstos sean mínimos, logrando así una mejor nutrición de la población infantil.

\section{Agradecimientos}

Se agradece al Hospital Nacional de Chiquimula, en especial al Comité de Docencia e Investigación por haber aprobado el tema de investigación y haber permitido realizar el trabajo de campo en el hospital en mención, así mismo, al departamento de Estadística por haber proporcionado los datos necesarios para el cálculo del universo y muestra. Un agradecimiento especial también a la Dra. María Sonia Capetillo Lera por su gran apoyo y haber sido una excelente guía durante la realización de la investigación.

\section{Referencias bibliográficas}

INCAP Instituto de Nutrición de Centro América y Panamá, Guatemala. (s.f.) Alimentación complementaria (en línea). Guatemala. 33 p. Consultado 18 ago. 2017. Disponible en http://www.incap. int/dmdocuments/inf-edu-alimnut-COR/temas/2.alimentacioncomplementaria/pdf/2.alimentacioncomplementaria.pdf

OMS Organización Mundial de la Salud, Suiza \& OPS (Organización Panamericana de la Salud, Washington). (c2010). La alimentación del lactante y del niño pequeño: capítulo modelo para libros de texto dirigidos a estudiantes de medicina y otras ciencias de la salud (en línea). Washington, D.C. 108 p. Consultado 18 ago. 2017. Disponible en http://apps.who.int/iris/bitstream/10665/44310/1/ 9789275330944_spa.pdf

UNICEF Fondo de las Naciones Unidas para la Infancia, Argentina. (2012). Evaluación del crecimiento de niños y niñas (en línea). Argentina. p. 43. Consultado 17 ago. 2017. Disponible en https:/www. unicef.org/argentina/spanish/Nutricion_24julio.pdf 


\section{Sobre la autora}

\section{Katherine Michelle Cuéllar Alas}

Médica y Cirujana egresada del Centro Universitario de Oriente CUNORI de la Universidad de San Carlos de Guatemala, la investigación de tesis de la cual escribió el artículo tiene como título "Caracterización del patrón alimenticio en niños menores de un año de edad. Actualmente se desempeña como médico residente en el Hospital Centro Médico de Chiquimula.

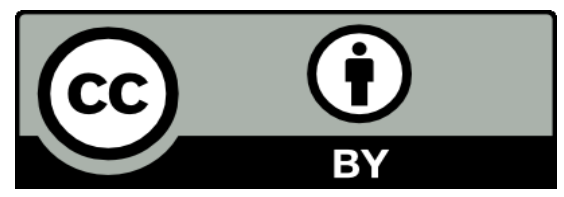

Este texto está protegido por una licencia CreativeCommons 4.0.

Usted es libre para compartir, copiar y redistribuir el material en cualquier medio o formato y adaptar el documento, remezclar, transformar y crear a partir del material para cualquier propósito, incluso comercialmente, siempre que cumpla la condición de atribución: usted debe reconocer el crédito de una obra de manera adecuada, proporcionar un enlace a la licencia, e indicar si se han realizado cambios. Puede hacerlo en cualquier forma razonable, pero no de forma tal que sugiera que tiene el apoyo del licenciante o lo recibe por el uso que hace. 